\title{
Creación de textos corpo-sonoros como otra forma de expresión estética*
}

\section{Creation of sound-corporeal texts as another form of aesthetic expression}

\author{
María José Velásquez Martínez ${ }^{1}$
}

Para citar este artículo: Velásquez, M. J. (2018). Creación de textos corpo-sonoros como otra forma de expresión estética. Infancias Imágenes, 18(1), 122-131

\section{Resumen}

La creación de textos corpo-sonoros surge como una propuesta investigativa cuya finalidad es expresar emociones y sentimientos que germinan de la historia de vida de cada participante del curso 301 de la jornada tarde en la I.E.D. Antonio Nariño, sede A. Para guiar este proceso de creación, nos preguntamos cómo potenciar la creación corpo-sonora para la exteriorización de emociones y sentimientos para así permitir otras formas de expresión estética, desarrollada por medio de actividades relacionadas con el conocimiento del cuerpo y la gran variedad de emociones que albergamos. Esto permite ampliar el vocabulario referente a las emociones, reconocer la potencia que es la historia de vida para crear e identificar al cuerpo como parte del ser que somos, para expresar de una forma estética, es decir, contemplando las producciones de los demás compañeros como una posibilidad de disfrute y gozo de lo creado a partir de la vida misma.

Palabras clave: expresión corporal, estética, relato, sentimiento.
Recibido: 22-octubre-2018 / Aprobado: 12-diciembre-2018

\begin{abstract}
The creation of sound-corporeal texts emerges as a research proposal whose purpose is to express emotions and feelings that sprout from the life story of each participant of the afternoon class 301, at the public school Antonio Nariño. To guide this creation process, we wondered how to enhance the sound-corporeal creation to externalize emotions and feelings in order to allow other forms of aesthetic expression, developed by means of activities related to the knowledge of the body and the great variety of emotions that we have. This allows expand the vocabulary concerning to emotions, to recognize the potency in life history to create and to identify the body as part of the being that we are, to express an aesthetic way, that is to say, contemplating the productions of other colleagues as a possibility of enjoyment and pleasure of what created from life itself.
\end{abstract}

Keywords: movement education, aesthetic, story, feeling.

\footnotetext{
* Investigación iniciada en agosto del 2016 y cuya fase de recolección de información finalizó en diciembre del 2017. En ella se entiende por texto corpo-sonoro toda secuencia rítmica creada a partir del uso intencionado del cuerpo como instrumento generador de sonidos.

1 Estudiante de la Licenciatura en Pedagogía Infantil de la Universidad Distrital Francisco José de Caldas. Correo electrónico: majovema11@
} hotmail.com 


\section{Introducción}

Este proyecto se desarrolló en el marco de la práctica formativa (2016-2017), inscrita a la línea de profundización de lenguaje, creación y comunicación de la Licenciatura en Pedagogía Infantil de la Universidad Distrital Francisco José de Caldas. Dicho proceso se llevó a cabo en el curso 301, en la jornada de la tarde de la Institución Educativa Distrital Antonio Nariño, sede A, la cual se ubica en el barrio Villa Luz, en la localidad de Engativá de la ciudad de Bogotá D. C.

Dicha población era de 23 niños y niñas, con edades comprendidas entre los 7 y los 10 años, donde las situaciones y composiciones familiares eran diversas. Por ejemplo: cuatro estudiantes tenían a sus padres juntos, a los restantes 19 se les había muerto algún padre de familia, fueron abandonados a sus abuelos o tíos, o sus padres estaban separados. Es importante recalcar esto debido a que la mayoría de historias de vida contadas durante el proceso de investigación venían relacionadas con las dificultades que cada uno enfrentaba hacia dichas ausencias y cómo buscaban en otro tipo de actividades y seres, como mascotas, una compañía y una forma de destacarse entre sus compañeros de clase.

Es necesario mencionar que se puede entender por el concepto de creación corpo-sonora aquellas secuencias rítmicas creadas a partir del cuerpo como instrumento y como parte de nuestro ser. El cual está atravesado por sentimientos y emociones que pueden ser exteriorizadas por medio del relato para manifestar las vivencias de los niños y las niñas, concibiéndose el cuerpo como "elemento/expresivo básico con que cuenta el ser humano para habitar e interactuar en el espacio y comunicarse con sus semejantes" (Plata, 2009, p. 14).

Esta investigación se realizó a partir del reconocimiento y la valoración de las acciones de los estudiantes respecto al trato con sus compañeros. Situación en la cual se evidenció que algunos estudiantes demostraban ser más participativos y tenían mayor desenvolvimiento al hablar de su diario vivir, mientras que otros se mostraban reacios, por lo que no hablaban de sus experiencias. Ello permitió deducir y evidenciar que algunos tenían actitudes dominantes sobre los otros al momento de la participación.
Por otro lado, también se notaba un gran interés por las actividades relacionadas con el cuerpo tales como bailar, jugar o realizar deportes, con una gran predilección por aquellas donde pudiesen realizar composiciones disponiendo el cuerpo en posiciones estilizadas, como en la gimnasia.

Una vez finalizada la fase inicial de inserción e interacción nos planteamos la siguiente pregunta de investigación: ¿cómo potenciar la creación corpo-sonora para la exteriorización de emociones y sentimientos que posibiliten otras formas de expresión? Teniendo en cuenta este interrogante, se orientaron las acciones con los niños y niñas del curso 301 de la IED Antonio Nariño, para promover el desarrollo de la corporeidad como forma de expresión a partir de la creación estética de textos corpo-sonoros a través de la exploración de sus emociones y sentimientos.

\section{Experiencia investigativa la creación} corpo-sonora como lenguaje expresivo Tomo como primer antecedente el trabajo de PeIlinski (2005), quien menciona cómo la práctica musical está implicada en la sensibilidad y la significación humana, permitiendo así la identificación e implicación con lo percibido, no solo desde lo musical, sino desde todas aquellas representaciones de orden estético que forman parte de la experiencia.

En este sentido, la estética musical del grupo con el cual se trabajó estaba dirigida principalmente hacia ritmos como el reguetón y el rap. Por ende, el grupo se sentía más identificado hacia las temáticas de orden sexual, problemáticas familiares mencionadas en las canciones de rap, además de los bailes que dan cuenta de los ritmos latinos.

De dichos aspectos podemos rescatar, para esta investigación, el valor de la experiencia sonora generada con el cuerpo. Pues, más allá del sonido en sí mismo, esta experiencia se refiere a la combinación de distintos ritmos resultantes de un proceso de exteriorización de sentimientos y emociones surgidos a partir de las distintas situaciones, primero de forma individual y luego en concordancia con el resto del grupo, viéndose esta como una forma de realizar terapia, expresión emocional e incluso llega a concebirse como un constructo social dado 
en el grupo de participantes tras el vínculo generado mediante la acción investigativa y práctica.

Así es como el sonido puede ser visto y trabajado desde nuestra investigación a partir de los elementos que constituyen las historias de vida y cómo ellas pueden ser representadas mediante secuencias rítmicas. Es necesario conocer del otro y de lo que le acontece para construir sociedad desde el aula misma, de manera que se puedan comunicar por medio de sus creaciones posibilitando de esta forma un ambiente fraterno para compartir experiencias que proporciona información de lo que le acontece al otro. Estas creaciones adquieren la dimensión estética cuando se configuran como encuentros reflexivos, pues según Greene: "Los encuentros reflexivos con las representaciones que marcan nuestros días nos permiten descubrir más y más de las formas artísticas particulares y de las maneras en que se elaboran" (2004, p. 29).

También se consultó como antecedente el trabajo de Bogoya, Galeano y Mendivelso (2016), quienes hablan acerca del sonido como un elemento de comunicación y de expresión el cual es utilizado por el lenguaje para transmitir información dado que muchas veces surge de los acontecimientos que pasan por la historia de cada sujeto. Esto se demuestra en los gustos musicales que se evidencian en cada generación, los cuales manifiestan parte de su propia construcción social, por lo tanto, tiene un sentido en cada espacio sociocultural del que los sujetos hacen parte.

Esta construcción sonora no es absolutamente estable, sino se va trasformando a través del tiempo, de la misma forma como se presentan nuevos acontecimientos para las personas; porque incluso lo que consideramos moda también cambia a través del tiempo.

Por último, al establecerse una relación de las personas con la música aprehendida por medio del contexto social, el cuerpo adquiere relevancia allí también al tener los medios para comunicarse, para expresar todo aquello que le acontece, permitiendo así la identificación con el mundo-contexto que le rodea.

Otro de los referentes utilizados fue Bohórquez (2016), quien toma el concepto de música como un "aspecto fundamental en la vida humana, se encuentra presente en todas las culturas, es una forma de expresión artística en la que se resalta la imaginación, creación e innovación" (p. 34); que es posible encontrar en el diario vivir, debido a que se concibe como una manifestación cultural en la que están en juego los atributos resaltados, todo ello teniendo en cuenta que las manifestaciones culturales son muy próximas a la cotidianidad de niños y niñas.

Es fundamental hablar de la combinación de elementos como lo son el cuerpo y el sonido para llegar a conformar lo que se denomina como la creación corpo-sonora, la cual se ha materializado en apuestas como Percuaction, considerada una de las organizaciones orientadas al ritmo y la percusión en el mundo, desde donde se concibe la percusión corporal como "ejercicios de carácter ritual los cuales, con el paso del tiempo, se convertirían en prácticas de expresión artística" (Mantilla, 2014, p. 4). Pero dichas prácticas se mantienen hasta el día de hoy y se pueden visibilizar como bailes y, en el contexto colombiano, bailes típicos de cada región que denotan una historia de las prácticas socioculturales.

Es importante resaltar los trabajos que se han hecho al interior de Colombia con organizaciones gubernamentales, artísticas y educativas en pro de la recopilación y documentación de memorias, trabajos con y para el cuerpo, alternativas para el afrontamiento y estrategias de resistencia para sobrellevar el dolor de la guerra que ha azotado miles de vidas en nuestro país.

Teniendo en cuenta lo anterior, el primer trabajo que debo mencionar es el del Centro Nacional de Memoria Histórica alrededor de los cuerpos usados en la guerra, violentados de forma sexual. El informe se llama La guerra inscrita en el cuerpo $(\mathrm{CNMH}$, 2017) y en él se puede observar la recolección de la información y de testimonios de mujeres víctimas de la violencia en sus propios cuerpos. Sin embargo, muestran las alternativas por las que ellas han optado para proteger a los suyos y superar el sufrimiento. Algunas de estas estrategias de afrontamiento que se señalan en el informe son: el silencio, la defensa del territorio-cuerpo, la espiritualidad y el erotismo; sobre todo este último, como una forma de reclamar su propio cuerpo y para volver a tener 
una vida placentera. Cabe resaltar que este es un informe que manifiesta lo acontecido y, por ello, en su introducción recalca:

Las víctimas de violencia sexual han vivido en carne propia las vejaciones que se ejercen sobre sus cuerpos considerados disponibles, reducibles a objetos; esta violencia que permea todos los espacios sociales. En sus cuerpos están impresas las marcas de una sociedad que silencia a las víctimas, de un Estado incapaz de hacer justicia, de familias y comunidades tolerantes a las violencias de género y de un manto de señalamiento, vergüenza y culpa que impide que se reconozca la verdad sobre lo sucedido. (CNMH, 2017, p. 11)

Una segunda experiencia para mencionar es la del Colegio del Cuerpo, fundada por Álvaro Restrepo y Marie France Delieuvin en Cartagena de Indias en 1997. Este colegio se creó como una "oportunidad de acercarse a la dimensión expresiva y artística del cuerpo humano, a través de la danza contemporánea" (Colegio del Cuerpo, s.f.). Este, más allá de ser un sitio en el que se aprende a danzar, es un sitio al que llegan muchos jóvenes víctimas de la violencia en la cual el cuerpo pierde su sentido sacro, deja de ser el cuerpo propio, aquel que siente, me pertenece y me hace ser, y pasa a ser desecho pues solo se considera materia y no ser.

Como una apuesta por obras teatrales para el festejo de la paz fue creada SacrifiXio: la consagración de la Paz, esta es una de sus últimas presentaciones en las que se hace "reflexión sobre ese esfuerzo gigantesco que hemos hecho, que ha hecho este gobierno, que estamos haciendo los colombianos por cambiar esta mentalidad, por encontrar otro lenguaje, por construir un proyecto colectivo de nación" (Mimbre, 2018). Con esta y muchas más obras teatrales y dancísticas realizadas ponen de manifiesto el trabajo que realizan con niños, niñas y jóvenes alrededor del cuerpo sacro y el rescate de este, sensibilización e investigación.

Finalmente, es necesario reconocer que "la música simboliza y expresa el principio de la comunicación mutua" (Dewhurst, 1993, p. 55), porque cuando nacemos, y aún en el vientre materno, estamos rodeados de sonidos que pueden llegar a conformar la música; pues el sonido es inherente a la naturaleza, al ser parte de ella, es inherente al ser humano.

\section{Metodología}

Esta investigación se realizó con un enfoque cualitativo con el cual tomamos una posición desde las propiedades del contexto, lo que nos permitirá centrarnos en aspectos más propios de cada persona, teniendo en cuenta su construcción desde los diferentes ámbitos que lo forjan. Tomando la postura de observadora participante en la investigación, se tuvieron en cuenta algunos elementos que menciona Cerda (1993), como lo son: abandonar las preconcepciones para explorar junto a los sujetos con los que se realiza la investigación, indagar acerca de lo que evidenciamos dentro del mismo contexto y establecerse desde él para entenderlo, no desde una mirada exterior a este, lo que no permite ver la realidad en total y desde donde se produce.

La modalidad desarrollada durante la investigación fue la de investigación-acción educativa. En esta, el conocimiento no surge para impartir, sino que nace en el proceso en el cual se enmarca y bajo unas acciones a realizar en un contexto educativo, adicionando la calidad del pensamiento que se genera teniendo presente que "la comprensión humana es la cualidad del pensamiento que se construye poco a poco en el proceso de aprendizaje" (Elliot, 2000, p. 84). Lo anterior, sin dejar de lado que el proceso de aprendizaje se da desde los estudiantes y los profesores en el quehacer docente con la oportunidad de evaluar las estrategias empleadas y la calidad del trabajo.

Esta modalidad nos permite la apertura a las diversas conjeturas que surgen desde una perspectiva teleológica. Es decir, con la intención de guiar el proceso hacia un fin donde predomina el diálogo ${ }^{2}$ se pudo tener una mayor claridad al entender las historias de vida y de dónde surge cada una.

Algunos de los procedimientos usados para la recolección de información fueron:

2 Acá lo he considerado como una de las intenciones más importantes para el desarrollo de la investigación realizada, pues se refuerzan las historias de vida. Esto no es solo por medio del diálogo con los niños y las niñas, sino también con la maestra titular, la cual lleva más tiempo en interacción con los infantes. 
- La observación participante, debido a que se estableció una relación de diálogo directo con los niños y las niñas para entenderlos desde su propio lenguaje y poder "conocer todos los aspectos y definiciones que posee cada individuo sobre la realidad y los constructos que organizan sus mundos" (Cerda, 1993, p. 244).

- La entrevista focalizada nos permitió indagar acerca de las concepciones que hay respecto a un tema en específico, desde las propias vivencias y emociones. Pero, para ello, debía existir un contacto previo con la población, un acercamiento y compenetración para que surgiera la comodidad y el ambiente para la socialización de la experiencia.

- Al ser una propuesta que genera productos usualmente efímeros, en los que los resultados se desvanecen apenas son realizados, se planteó la necesidad de constatar los resultados, creaciones, por medio del registro audiovisual que nos permitió tener la experiencia al alcance y con alternativas como la bitácora o diario de campo, con lo que se pudo observar desde las categorías propuestas al interior de la investigación.

\section{La expresión corporal y sonora para una creación estética}

La expresión corporal fue un componente esencial en esta investigación debido a que nos hizo pensar acerca del sentido de la corporalidad en la escuela. En esta perspectiva, es necesario saber cómo potenciarla, ya que podemos decir que no es un concepto ni una práctica que se trabaje a profundidad en la escuela la cual debería ser un escenario para la definición de la autonomía para finalmente vivir en libertad (Corral, 2009). Por el contrario, se evidenció que se hacía uso del cuerpo para pasar de un momento a otro dentro del aula, es decir, se especificaba el manejo del cuerpo para estiramientos y así pasar de una materia a otra.

Por lo tanto, el cuerpo no representaba más que un medio para el estudio, una forma de estar en el aula y memorizar, lo que deja de lado el sentido del cuerpo por el cuerpo. Es decir, el cuerpo que por la experiencia que vive la persona puede comunicarse sin necesidad de la palabra al estar el cuerpo físico y sensorial "habrá que advertir aquí que el cuerpo, considerado en cuando a lo sensitivo, o lo estiológico, depende del cuerpo material entendido como aparición y miembro del mundo circundante personal" (Illescas, 2014, p. 25). De esta manera podemos entender el cuerpo que comunica como materia y el cuerpo que está y vive en el mundo, somos cuerpo físico y sensación, emoción y sentimiento.

Como una forma de reconocimiento de las emociones que nos embargan se les ha brindado a los niños y a las niñas un listado de varias de ellas y se les ha pedido que escogiesen un mínimo de tres y luego las plasmaran por medio de un dibujo en las hojas blancas entregadas. Con esta actividad se han identificado algunas emociones generales como lo son la felicidad, ira, sorpresa y enfado. Sin embargo, con la intención de ampliar el vocabulario y de reconocer que existen más, se les ha pedido que escogiesen distintas a las de los otros compañeros. Tras esta actividad han surgido algunas emociones más que clasificaría como principales para los alumnos del curso: la soledad, la tristeza y la angustia. Lo particular es que dichas emociones se pueden clasificar en emociones negativas y son las que rondaban tanto en esta actividad como en la siguiente, que es la de los mapas corporales.

Paralelamente a las actividades planteadas en la secuencia didáctica se hicieron ejercicios relacionados al movimiento, el cuerpo propio y la empatía. Se les pedía que caminaran por el salón y que dependiendo las palmas que se tocaran fuesen más rápido o más despacio, todo ello teniendo en cuenta el espacio de sus otros compañeros. Luego se les pedía que hicieran reconocimiento del cuerpo de otro compañero con el cual no tuviesen mucho contacto por lo general, hallar qué puntos de su cuerpo les molestaba, cuáles eran los que más les gustaba y sus características físicas principales. A este punto debo agregar que también empezaron a hacer por cuenta propia movimientos con los cuerpos de los demás para saber hasta qué límite podían llegar respecto a flexibilidad y resistencia en alguna posición.

El trabajo con mapas corporales constituyó una potencia para el reconocimiento de las emociones desde el propio cuerpo de las y los participantes. 


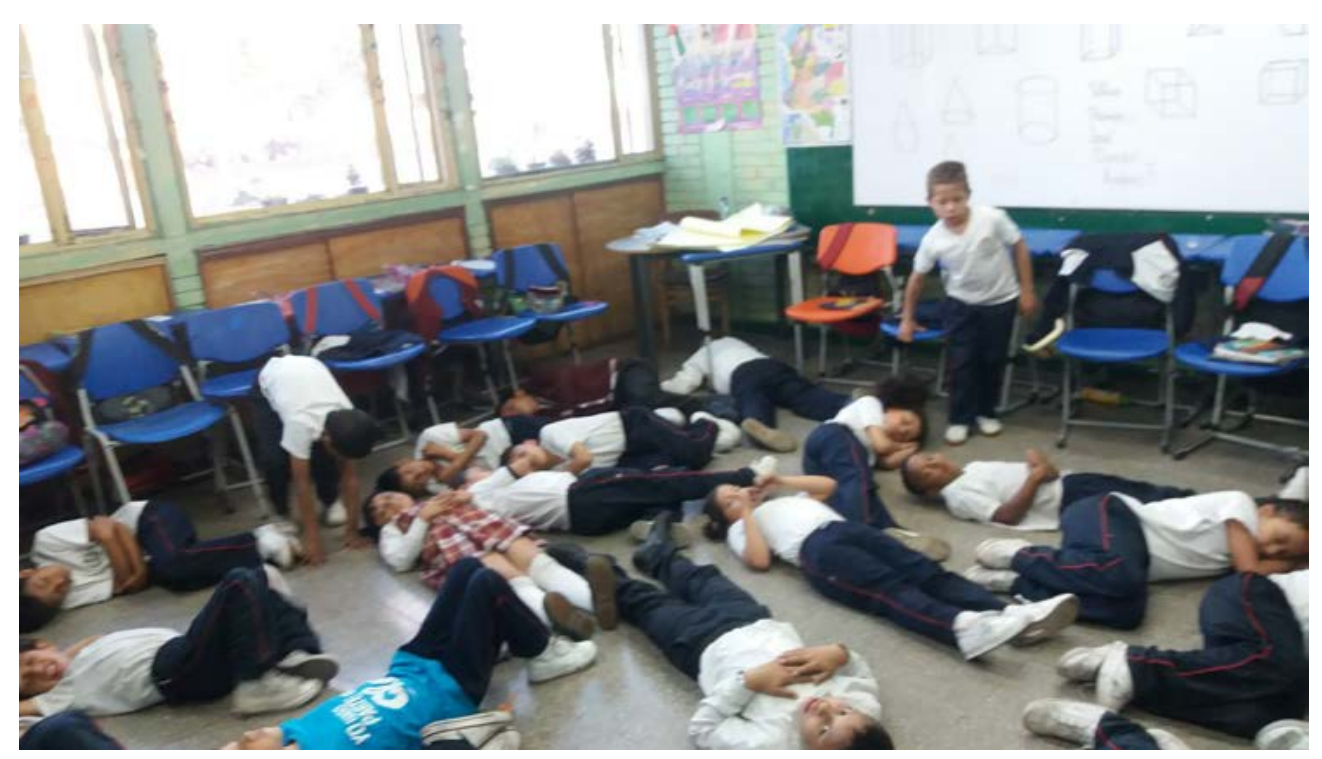

Figura 1. Reconocimiento del cuerpo propio.

Fuente: elaboración propia de la autora.

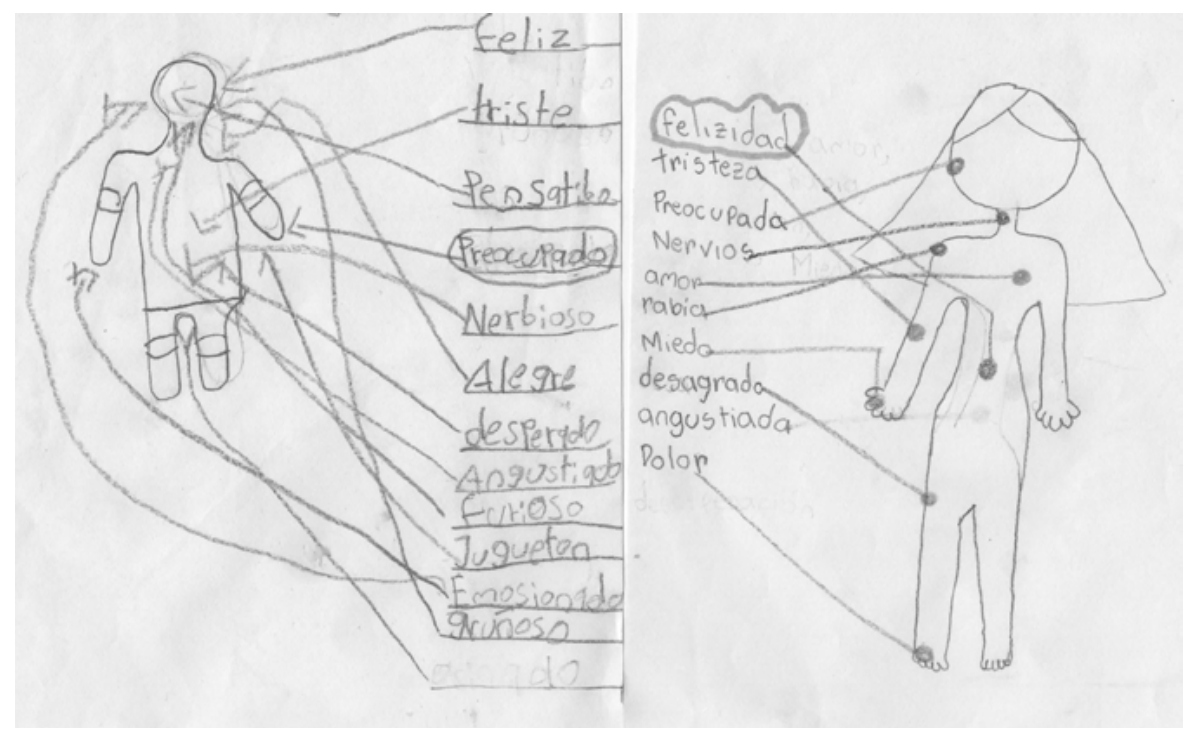

Figura 2. Mapa corporal de sentimientos y emociones.

Fuente: Fotografía de la autora a los dibujos de dos de los participantes.

Pues permite visualizar las emociones que de allí surgían; siendo así posible tener memoria de una emoción, pero también de una historia específica surgida a partir de situaciones concretas que vivían los niños y las niñas, al igual que todas las personas, ya que por nuestro cuerpo y emocionalidad pasan todas las situaciones.
El uso de mapas corporales en los que los niños y las niñas relacionaban los sentimientos y emociones con la parte del cuerpo donde las podían identificar sirvió como punto de partida para localizar aquellas partes en las cuales ellos y ellas pensaban que tenían mayor relevancia sus experiencias diarias. Así, se vincula la historia de vida, 
no solo oral sino también escrita, ya que, como plantea Aceves (1998), "lo que importa es la experiencia y trayectoria de vida de tal sujeto y no, particularmente, un tema concreto de indagación" (p. 211). Aunque con un eje determinado, como la creación corpo-sonora, la historia de vida permite evidenciar el acontecer diario de los niños y las niñas y cómo este va cambiando mediante las experiencias, la medida en que los enriquece como sujetos sensibles.

La sensibilidad adquirida hacia cada uno de los compañeros a partir de esta actividad, ya que voluntariamente algunos han pasado a explicar su mapa corporal, ha puesto de manifiesto la capacidad de conocernos mutuamente a partir de la escucha. Además, ha sido interesante evidenciar cómo los niños y niñas dan luces de dónde puede estar sintiendo su compañero alguna emoción con el trascurrir de las sesiones. También se notó que la preocupación por conocer la vida y cómo esta le afecta en su cuerpo ha hecho que su diálogo muchas veces fuese más profundo que el responder "estoy bien". Por el contrario, con el conocimiento de cómo las emociones afectan cada parte del cuerpo ellos pudieron relacionar el cuerpo como sensación y emoción más allá de materia.

A continuación, se generó un proceso de descubrimiento del sonido en relación con la emoción e historia de vida que lo originó. Es decir, se estuvo trabajando alrededor de la memoria del suceso, cómo ellos creían que sonaba y en qué parte del cuerpo. Lo interesante a destacar de este proceso es la ayuda de los demás compañeros para la creación del sonido y su ritmo a partir de lo que habían escuchado de las historias y cómo ellas afectaban a sus compañeros. Fue desde ahí que comenzó el proceso grupal realmente, al preocuparse los unos por los otros.

Teniendo como precedente que "el sonido es una parte integral de nuestras vidas. Los seres humanos han hecho uso del sonido desde sus tiempos más remotos para obtener información sobre el mundo que los rodea y para comunicarse entre sí" (Dewhurst, 1993, p. 14), fue necesario empezar a mostrar ejemplos de forma audiovisual que evidenciaran la creación sonora por medio del cuerpo.
Un ejemplo de lo anteriormente es Túpac Mantilla (2014), del cual se pueden encontrar videos de sus creaciones e interpretaciones en la internet ${ }^{3}$.

Es importante señalar que en el curso en el cual se realizó la investigación, en concreto las clases de educación artística, los estudiantes exploraron también con la creación de sonidos a partir del cuerpo. Sin embargo, es necesario diferenciar el que la creación corpo-sonora propuesta en esta investigación era de orden expresiva y estética, con base en los sentimientos y emociones. Es decir, en lo sensorial y lo cognitivo como dimensiones a través de las cuales, según lo planteado por Finol (2001), se puedan reconocer distintas "formas de relación con la realidad — sea esta física o biológica, cultural o espiritual-, que originan un conocimiento, una experiencia que, en la tradición hedonista griega, permite encontrar placer y satisfacción en las cosas" (p. 2). Así se puede describir lo estético como el producto entre lo sensible o perceptivo y lo real, cultural, contextual. No se limita a lo que tenemos que hacer por obligación, sino también al goce de dichas experiencias.

Para finalizar el trabajo con los niños y las niñas, se procedió a nombrar nuestra creación donde algunos daban ideas del nombre con el que se quería nombrar como cuerpos en ruido, corpo-sonoro e historias musicales así que se realizó una votación y la creación tiene por nombre historias musicales. Al preguntar el porqué de este nombre el niño que ha dado la idea mencionó:

[...] de cada historia hemos puesto un sonido. Ahora todos hicimos música con todas nuestras historias ${ }^{4}$.

Esta ha sido otro de los elementos a resaltar y es el sentido de pertenencia de la historia, puesto que es la propia y de ahí creé, nadie más pudo haberlo hecho sino yo mismo y con la ayuda de los compañeros como se puede ver en el video del resultado final. ${ }^{5}$

3 Muestra de algunas de sus interpretaciones se pueden encontrar en el siguiente vínculo. Dicha demostración es realizada por Túpac Mantilla y Sofía Rei. Véase: https://www.youtube.com/ watch?v=y4U7cdUoxN4

4 Charla abierta con los niños y niñas del curso el 28 de noviembre del 2017.

5 Video final de la creación corpo-sonora. Véase: https://www.you tube.com/watch? $=$ =MlopXZwAe1I 
Como parte de la valoración de la experiencia estética también resaltamos la contemplación. Manejada en este proceso investigativo desde la individualidad, desde lo que cada persona sentía tras presentar su historia de vida al ámbito grupal, pues no solo veíamos las representaciones de las historias de vida, sino que también podíamos visualizar el producto final de cada compañero, los sentimientos y emociones reflejados en los sonidos con el cuerpo para el ensamblaje de la creación corpo-sonora. Esto para así lograr la unión de los sonidos para la creación de una composición que ampliara las posibilidades expresivas hacia nuevos horizontes. Pues, con Greene, creemos que "el abrir ventanas y puertas para la gente, el soltarla para usar la imaginación, sus mentes y sus capacidades perceptivas, puede salvar vidas al igual que cambiarlas" (2004, p. 60).

\section{Conclusiones}

Menciona Corral "el cuerpo define su autonomía y va aprendiendo a pensar y a vivir en libertad" (2009, p. 22). Sin embargo, este proceso se ha dificultado. Es evidente que para llegar a ese aprendizaje corporal de la libertad es necesario tiempo y menos agentes reguladores de lo que está bien o mal en la escuela; no obstante, sabemos que son mecanismos implantados a lo largo de los años al pensarse la educación como un acto de poder y dominación, educación como un acto de implantar las formas de estar que se creen son buenas.

La creación corpo-sonora, más allá de una investigación, ha surgido como un espacio de expresión y exteriorización de las emociones y sentimientos que surgen de la historia de vida y es allí donde la podemos diferenciar de la creación de sonidos con el cuerpo; pues las superficies pueden resonar, pero en este caso, resuena la historia y las emociones de cada uno de los participantes. Más allá del sonido está lo que significa y el trabajo de recuperación de las historias; son estas las que nos definen y nos potencian para ser creadores.

Ha sido dificultoso el lograr que los niños y las niñas hagan movimientos desde su propio sentir, pues muchas veces hay un ente regulador de sus movimientos. "Las representaciones sociales le asignan al cuerpo una posición determinada dentro del simbolismo general de la sociedad. Sirven para nombrar las diferentes partes que lo componen y las funciones que cumplen, hacen explícitas sus relaciones" (Le Bretón, 2002, p. 13). Estas regulaciones se hacen desde las instituciones por donde pasan los niños y las niñas; la familia, el colegio, el contexto en general, el cual es el espacio donde ellos se desenvuelven, se comunican y, de alguna u otra forma, en sus relaciones crean espacios de compartir, como en los descansos y tiempos libres.

Desde un sentido utilitarista del cuerpo este era mencionado por los niños y las niñas como la materia de la cual estamos compuestos en el sentido orgánico, ya que mencionaban:

[...] son las partes internas como: el corazón, los pulmones y también están las externas como las piernas... ah que son el sistema locomotor ${ }^{6}$.

Al plantearse la idea de un cuerpo que puede expresar lo que somos y el camino que llevamos, el horizonte del significado del cuerpo se amplió. De lo dicho anteriormente, podemos decir que, por medio de la indagación, con preguntas y a través de un procedimiento dialógico, se pueden cambiar las concepciones que tenemos, incluso, de nosotros mismos, pues con el tiempo la idea del cuerpo orgánico fue cambiando a una de cuerpo sensorial, una idea desde la corporeidad donde se pueda:

Hacer del cuerpo un instrumento perfecto de adaptación del individuo a su medio tanto físico como social, gracias a la adquisición de la destreza que consiste en ejecutar con precisión el gesto adecuado en cualquier caso particular y que se puede definir como el dominio fisiológico para la adaptación a una situación dada. (Le Boulch, 1993, p. 95).

La creación realizada da cuenta de dos tipos de expresión unidos: el corporal y el sonoro que permiten expresar los sentimientos y emociones que son inherentes a la humanidad y que fueron compartidas constantemente en el tiempo de trabajo con los niños y las niñas.

En este espacio también se evidenciaron aprendizajes emergentes del proceso como el respeto

$6 \quad$ Charla en el aula de clase del 14 de septiembre del 2017. 
por el otro, por su cuerpo, por su historia de vida; debido a que por medio del relato se han podido conocer más a fondo y así establecer lazos de empatía entre ellos. Así es como definimos la historia de vida como el potenciador de la creación.

Para finalizar, es necesario que como pedagogos, profesores, personas que trabajamos con la infancia, creemos espacios de expresión auténtica que correspondan al interés real de los niños y las niñas. Es decir, no con las limitaciones que usualmente se ponen como la plantilla, sino con la vida misma como interés. Muchas veces la expresión de los niños y las niñas se cohíbe tras no considerarla como esencial. Sin embargo, sus vidas salen a la luz de la realidad mediante sus comportamientos, historias y creaciones artísticas, compartidas algunas veces a sus compañeros por el vínculo y entendimiento que se dan entre algunos de ellos. Nuevamente se reafirma que la búsqueda social se da de acuerdo con similitudes estéticas para el goce y la satisfacción de lo que hacen o producen.

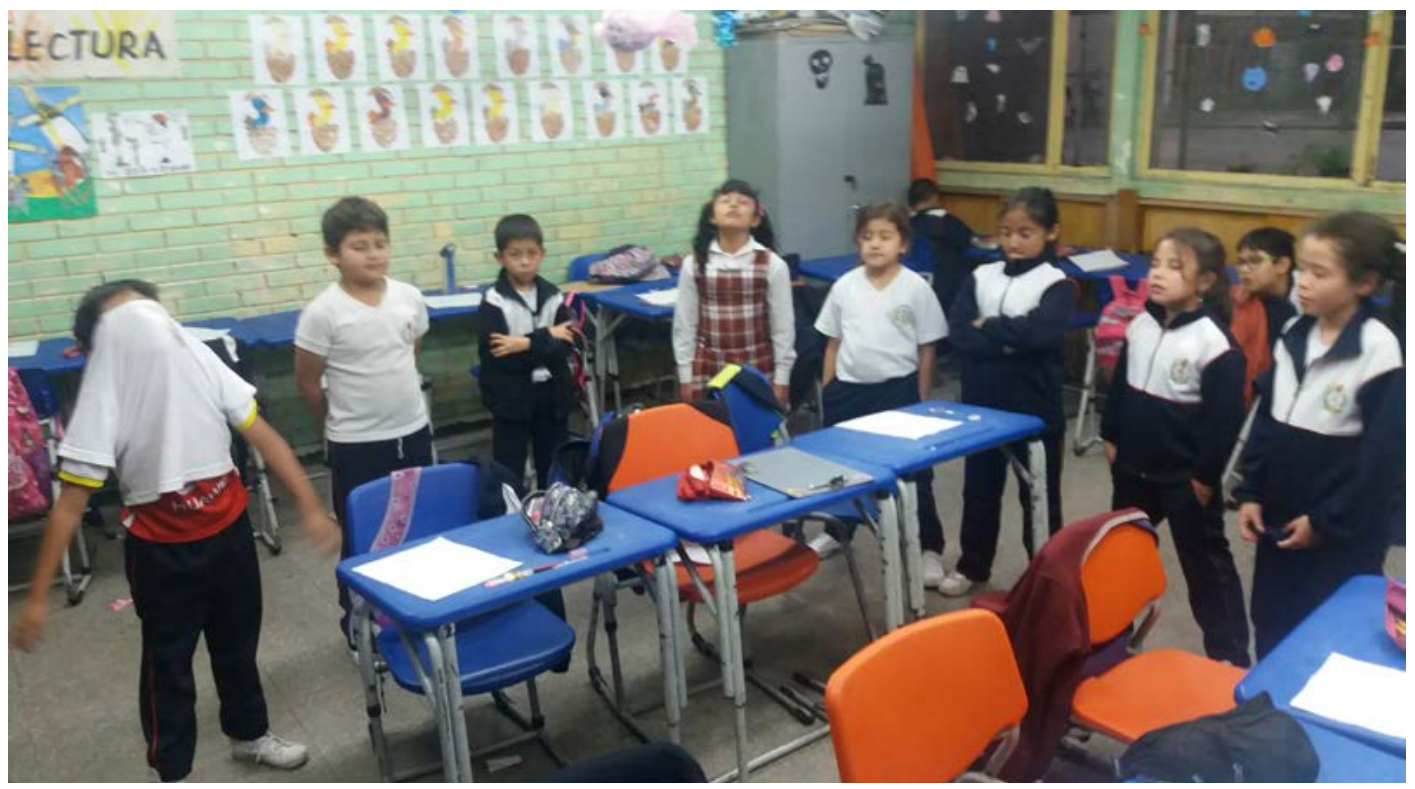

Figura 3. Mapa corporal de sentimientos y emociones.

Fuente: elaboración propia de la autora con permiso de los padres.

\section{Referencias}

Aceves, J. (1998) La historia oral y de vida: del recurso técnico a la experiencia de investigación. En J. Galindo (ed.), Técnicas de investigación en sociedad, cultura y comunicación (pp. 207276). México: Pearson Educación.

Bogoya, G.; Galeano, R. y Mendivelso, Y. (2016). La música y su incidencia en los saberes que construye la infancia sobre género y cuerpo. Universidad Distrital Francisco José de Caldas, Bogotá, Colombia. Recuperado de http://hdl. handle.net/11349/3871
Bohórquez, J. C. (2016). La canción como estrategia didáctica para desarrollar la comprensión lectora en el ciclo II (Trabajo de grado de maestría). Universidad Distrital Francisco José de Caldas, Bogotá. Colombia. Recuperado de http://hdl. handle.net/11349/3402

Centro Nacional de Memoria Histórica. (2017). La guerra inscrita en el cuerpo: informe nacional sobre violencia sexual en el conflicto armado. Bogotá: Centro Nacional de Memoria Histórica. Recuperado de http://www.centrodememoriahistorica.gov.co/descargas/ 
informes-accesibles/guerra-inscrita-en-el-cuerpo_accesible.pdf

Cerda, H. (1993). Los elementos de la investigación. Como reconocerlos, diseñarlos y construirlos. Quito: Abya Yala.

Colegio del Cuerpo. (s.f.). Colegio del Cuerpo. Recuperado de https://elcolegiodelcuerpo.org/en/ el-colegio-del-cuerpo/

Corral, M. (2009). Cuerpo, comunicación y sensibilidad. México D.F.: UACM.

Dewhurst, O. (1993). El libro de la terapia del sonido. Madrid: Edaf.

Elliot, J. (2000). La investigación acción en educación. Madrid: Morata.

Finol, J. (2001). Estética del cuerpo: esbozo de un análisis semio-antropológico. Arte y Ciencia: Buscando la belleza. Conferencia inaugural en la VIII Jornada de Odontología. Maracaibo, Venezuela: Universidad de Zulia.

Greene, M. (2004). Variaciones sobre una guitarra azul. Conferencias de educación estética. México D. F.: Edere.

Illescas, M. (2014). La vivencia del cuerpo propio en la fenomenología de Edmund Husserl. En A.
Xolocotzi y R. Gibu (eds.), Fenomenología del cuerpo y hermenéutica de la corporeidad (pp. 15-34). México D. F.: Plaza y Valdés.

Le Boulch, J. (1993). Psicocinética, educación y "APS" (actividad física y deportiva). En Memorias del I Congreso Argentino de Educación Física y Ciencias. La Plata, Argentina: Universidad Nacional de La Plata. Departamento de Educación Física, Facultad de Humanidades y ciencias de la Educación.

Mantilla, T. (2014) Percusión corporal. Vol. I. Currículo para la excelencia académica y la formación integral 40x40. Percuaction.

Mimbre (productor). (2018). 'SacrifiXio: la consagración de la Paz': la obra teatral que rinde tributo a los diálogos de paz. Recuperado de https://www.youtube.com/watch?v=avecj9Tb9z4

Pellinski, R. (2005). Corporeidad y experiencia musical. Revista Transcultural de Música 9. Recuperado de https://www.sibetrans.com/trans/articulo/177/ corporeidad-y-experiencia-musical

Plata, H. (2009). Anibailando. La danza zoomorfa 131 en la escena escolar. Bogotá: Gente Nueva. 